\title{
Evolution of the color indices in SN 2006aj associated with GRB 060218
}

\author{
V. Šimon ${ }^{1}$, G. Pizzichini ${ }^{2}$, and R. Hudec ${ }^{1}$ \\ 1 Astronomical Institute, Academy of Sciences of the Czech Republic, 25165 Ondřejov, Czech Republic \\ e-mail: simon@asu.cas.cz \\ 2 INAF/IASF Bologna, via Gobetti 101, 40129 Bologna, Italy
}

Received 19 March 2010 / Accepted 22 July 2010

\begin{abstract}
Aims. We analyze the photometric properties of SN 2006aj associated with GRB 060218. We also investigate this event in relation to other supernovae.

Methods. We determined the color indices from multiband photometry and investigated their time evolution by taking their time stretch factors into account. We used both the ground-based and UVOT/Swift data to ensure a coverage from the cosmic UV region

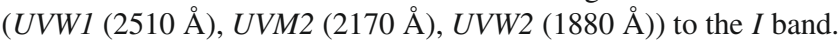

Results. We find a large difference between the values and time evolution of the color indices of the early phase $\left(t-T_{0} \leq 2.5 \mathrm{~d}\right)$ of the optical afterglow (OA) and the subsequent SN 2006aj. The clustering and small evolution of most color indices in UV in $t-T_{0} \leq 2.5 \mathrm{~d}$ resemble the behavior seen in the ensemble of OAs of long GRBs. In our interpretation, the light of the early OA comes from far above the photosphere of the progenitor and its wind. The outer layers of SN 2006aj underwent the strongest evolution among the considered supernovae of various types. We find that the adjustment of time evolution of various color indices by stretching is very helpful in searching for the proper supernova type. We attribute this evolution of SN 2006aj to the exceptionally low mass of its ejecta and the structural changes of the region producing the GRB. In our interpretation, we observed the different regions simultaneously in the complicated, asymmetric shape of SN 2006aj. The colors in the UV band can be explained if line blanketing undergoes only small variations and the UV emitting area tends to shrink during the evolution of SN 2006aj over the time interval investigated. Our approach is also of general importance for investigating supernovae in OAs.
\end{abstract}

Key words. gamma-ray burst: general - radiation mechanisms: non-thermal - ISM: jets and outflows - galaxies: ISM supernovae: general - supernovae: individual: SN 2006aj

\section{Introduction}

Color indices have been widely used in the study of many phenomena in astrophysics. For the study of optical afterglows (OAs) of GRBs, however, this powerful method is only very rarely applied, therefore in this field it is still quite innovative. Color indices enable us to resolve small variations in the spectral profile of OAs, using the measurements in the commonly used UBVRI filters. This method proved to be very useful for analyzing the common properties of OAs (Šimon et al. 2001, 2004a). For example, it enables us to distinguish among the individual radiation mechanisms. It also allows us to constrain the properties of the local interstellar medium of GRBs inside their host galaxies. The use of broad-band colors has been advocated for identifying young type $\mathrm{Ib} / \mathrm{c}$ supernovae ( $\mathrm{SNe}$ ) by Gal-Yam et al. (2004).

GRB 060218 is a remarkably long GRB (Cusumano et al. 2006; Campana et al. 2006), since it is located at redshift $z$ of only 0.03345 (e.g. Mirabal et al. 2006). This makes it the second nearest known GRB and thus allows a detailed study. It is associated with SN 2006aj. The study of the evolution of the light curves in the UBVRI bands was performed by Mirabal et al. (2006). The evolution of the infrared emission in the $J H K_{\mathrm{s}}$ bands including the color indices of this $\mathrm{SN}$ is presented by Kocevski et al. (2007). They argue that the lack of significant color change during the rise of this SN suggests little or no spectral evolution over the first 10 days in near infrared.
According to Campana et al. (2006), the early emission $\left(<10^{5} \mathrm{~s}\right)$ of this event is caused by the shock breakout in a Wolf-Rayet star progenitor. In contrast, Ghisellini et al. (2007) and $\mathrm{Li}$ (2008) interpret it as a synchrotron emission from a jet. Asymmetric explosion of SN 2006aj was advocated by Maeda et al. (2007), which was thus a similar case to SN 1998bw (Maeda et al. 2006). Mazzali et al. (2006) argue that the initial mass of the progenitor of SN 2006aj was only $20 M_{\odot}$. SN 2006aj possessed considerably less massive ejecta $\left(M_{\mathrm{ej}} \approx 2 M_{\odot}\right)$ then other $\mathrm{SNe}$ associated with GRBs $\left(M_{\mathrm{ej}} \approx 10 M_{\odot}\right)$. Also the kinetic energy of its explosion was about an order of magnitude weaker. This was the cause of its faster evolution. Pian et al. (2006) show that the luminosity and velocity of the ejecta of SN 2006aj are midway between SNe associated with GRB and those lacking such an association. They further argue that the GRB of SN 2006aj was less luminous and softer than the "classical" GRBs. Furthermore, it was an intrinsically weak and soft event rather than a classical GRB observed off-axis. Maeda et al. (2007) argue that the remnant should be a neutron star, not a black hole. The SN-GRB connection thus extends to a considerably broader range of stellar masses than previously thought.

In this paper, we apply the method of the color indices to the optical afterglow (OA) associated with GRB 060218. We search for the common properties of this event and other $\mathrm{SNe}$, particularly those associated with GRBs. A preliminary version of part of this analysis was presented by Šimon et al. (2009, 2010). 

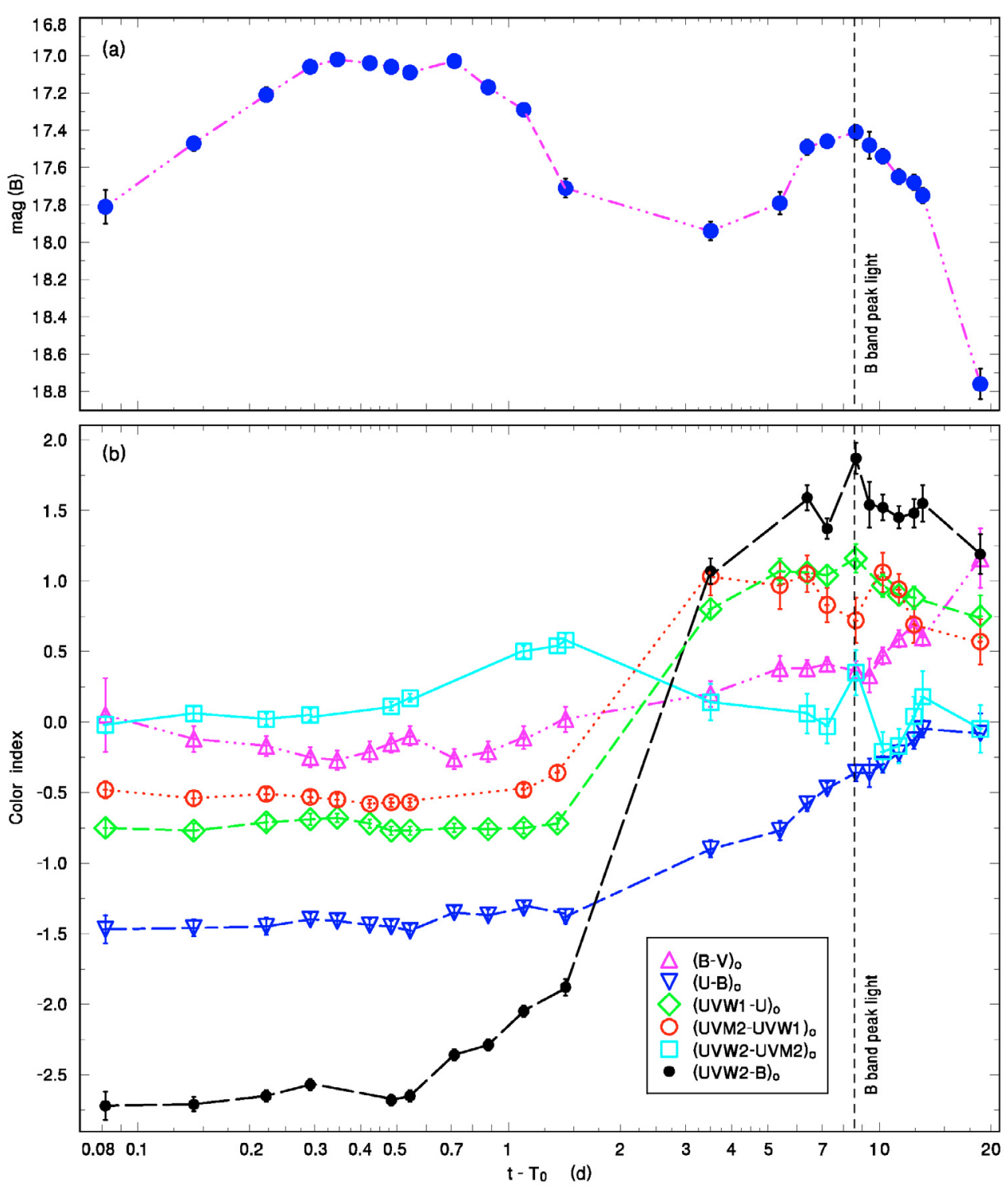

Fig. 1. The $B$ band light curve a) and the time evolution, in the observer frame, of the color indices b) of the $\mathrm{OA}$ of GRB 060218 (UVOT data). The points are connected by lines for convenience and may not precisely reflect the complicated profile of the variations in the data gaps. The data were corrected for the reddening and light contribution of the host galaxy as described in Sect. 3. (This figure is available in color in electronic form.)

\section{Observations}

The data used for our analysis of the OA of GRB 060218 are the ground-based photometric observations by Mirabal et al. (2006) and the data obtained by UVOT onboard Swift (http: // swift.gsfc.nasa.gov) (Gehrels 2004). UVOT observations used here were originally presented by Campana et al. (2006) and updated to the latest UVOT calibration by Brown et al. (2007). We make use of the electronic files of the UVOT data, which were provided to us by Brown et al. UVOT data were used for investigating the initial phase of the event during $t-T_{0}$ less than about $2 \mathrm{~d}$, where $T_{0}$ is the time of the GRB trigger. UVOT data also served the extension of the spectral coverage to the cosmic UV band where the observations by the ground-based telescopes are impossible. The data by Mirabal et al. (2006) overlap with UVOT observations in the $U B V$ bands. The extension of the observations to the infrared region $\left(J H K_{\mathrm{s}}\right)$ was secured by the data of Kocevski et al. (2007).

To help place the photometric properties of the late phase of the OA $\left(t-T_{0}>3.5 \mathrm{~d}\right)$ of GRB 060218 in context, the UVBRIJHK photometric data of SN 2002ap by
Yoshii et al. (2003) and the UBVRI observations of SN 1998bw by Galama et al. (1998) were included in our analysis.

\section{Data analysis}

The light curve of the OA of GRB 060218 used for our analysis is displayed in Fig. 1a. Two bumps are distinguished in it. The proper terminology has to be used here when referring to its parts. In the usual notation, the optical counterparts of GRBs are abbreviated as OAs no matter what the profiles of their light curves are. The brightness of this optical emission usually displays a peak shortly after the GRB followed by a power-law decay. In some OAs, another, fainter peak is also observed in the later phase (e.g. Bloom et al. 1999). The ratio of the heights of these peaks varies widely for the individual OAs. In this notation, the OA of GRB 060218 can be considered as the extreme case with two peaks of comparable heights. We divide the light curve of the OA of GRB 060218 into two parts: Phases I $\left(t-T_{0} \leq 2.5 \mathrm{~d}\right)$ and II $\left(t-T_{0}>2.5 \mathrm{~d}\right)$. Each part thus contains one peak.

UVOT contains six photometric filters $(V, B, U, U V W 1$ (2510 $\AA$ ), UVM2 (2170 $)$, and $U V W 2(1880 \AA))$ covering the 
Table 1. $B$ band magnitudes and interpolated color indices of the OA of GRB 060218, determined from UVOT observations.

\begin{tabular}{|c|c|c|c|c|c|c|c|c|c|c|c|c|c|c|}
\hline$t-T_{0}$ & $\operatorname{mag}(B)$ & $\sigma$ & $B-V$ & $\sigma$ & $U-B$ & $\sigma$ & $U V W 1-U$ & $\sigma$ & $U V M 2-U V W 1$ & $\sigma$ & $U V W 2-U V M 2$ & $\sigma$ & $U V W 2-B$ & $\sigma$ \\
\hline 0.0217 & & & & & & & -0.17 & 0.11 & -0.06 & 0.13 & -0.16 & 0.12 & & \\
\hline 0.0817 & 18.26 & 0.09 & 0.21 & 0.26 & -1.27 & 0.10 & -0.41 & 0.06 & -0.08 & 0.06 & -0.28 & 0.06 & -2.04 & 0.10 \\
\hline 0.1415 & 17.96 & 0.04 & 0.06 & 0.09 & -1.29 & 0.06 & -0.43 & 0.05 & -0.14 & 0.05 & -0.21 & 0.05 & -2.07 & 0.05 \\
\hline 0.2217 & 17.72 & 0.04 & 0.00 & 0.07 & -1.29 & 0.06 & -0.38 & 0.05 & -0.11 & 0.04 & -0.25 & 0.04 & -2.03 & 0.04 \\
\hline 0.2914 & 17.59 & 0.03 & -0.07 & 0.07 & -1.26 & 0.04 & -0.36 & 0.04 & -0.13 & 0.04 & -0.22 & 0.04 & -1.97 & 0.04 \\
\hline 0.3448 & 17.55 & 0.03 & -0.09 & 0.07 & -1.27 & 0.04 & -0.35 & 0.03 & -0.15 & 0.04 & & & & \\
\hline 0.4224 & 17.57 & 0.03 & -0.03 & 0.07 & -1.30 & 0.04 & -0.39 & 0.03 & -0.18 & 0.03 & & & & \\
\hline 0.4822 & 17.59 & 0.03 & 0.02 & 0.07 & -1.31 & 0.04 & -0.44 & 0.03 & -0.17 & 0.03 & -0.16 & 0.03 & -2.08 & 0.04 \\
\hline 0.5417 & 17.61 & 0.03 & 0.06 & 0.07 & -1.33 & 0.04 & -0.44 & 0.03 & -0.17 & 0.03 & -0.10 & 0.03 & -2.04 & 0.04 \\
\hline 0.7128 & 17.56 & 0.03 & -0.08 & 0.07 & -1.21 & 0.04 & -0.42 & 0.03 & & & & & -1.76 & 0.04 \\
\hline 0.8807 & 17.69 & 0.03 & -0.03 & 0.07 & -1.22 & 0.04 & -0.43 & 0.04 & & & & & -1.68 & 0.04 \\
\hline 1.0972 & 17.80 & 0.03 & 0.06 & 0.08 & -1.17 & 0.04 & -0.41 & 0.04 & -0.08 & 0.04 & 0.23 & 0.04 & -1.43 & 0.04 \\
\hline 1.3526 & & & & & & & -0.39 & 0.04 & 0.04 & 0.05 & 0.27 & 0.05 & & \\
\hline 1.4232 & 18.17 & 0.05 & 0.18 & 0.09 & -1.19 & 0.05 & & & & & 0.31 & 0.05 & -1.22 & 0.06 \\
\hline 3.5017 & 18.37 & 0.05 & 0.34 & 0.09 & -0.71 & 0.06 & 1.05 & 0.07 & 1.14 & 0.13 & -0.07 & 0.13 & 1.41 & 0.09 \\
\hline 5.3817 & 18.24 & 0.06 & 0.49 & 0.09 & -0.60 & 0.07 & 1.28 & 0.09 & 1.05 & 0.17 & & & & \\
\hline 6.3817 & 17.98 & 0.04 & 0.50 & 0.06 & -0.44 & 0.05 & 1.28 & 0.07 & 1.13 & 0.13 & -0.12 & 0.14 & 1.85 & 0.09 \\
\hline 7.2217 & 17.95 & 0.03 & 0.53 & 0.05 & -0.34 & 0.04 & 1.26 & 0.07 & 0.97 & 0.12 & -0.19 & 0.12 & 1.70 & 0.07 \\
\hline 8.6317 & 17.91 & 0.04 & 0.49 & 0.07 & -0.24 & 0.06 & 1.35 & 0.10 & 0.87 & 0.16 & 0.08 & 0.16 & 2.06 & 0.11 \\
\hline 9.3917 & 17.97 & 0.07 & 0.46 & 0.12 & -0.24 & 0.10 & & & & & & & 1.82 & 0.16 \\
\hline 10.202 & 18.02 & 0.04 & 0.58 & 0.06 & -0.17 & 0.06 & 1.17 & 0.08 & 1.08 & 0.14 & -0.28 & 0.14 & 1.80 & 0.09 \\
\hline 11.262 & 18.12 & 0.04 & 0.69 & 0.06 & -0.10 & 0.06 & 1.10 & 0.06 & 0.98 & 0.11 & -0.25 & 0.12 & 1.73 & 0.08 \\
\hline 12.382 & 18.15 & 0.04 & 0.78 & 0.06 & -0.02 & 0.06 & 1.07 & 0.08 & 0.81 & 0.13 & -0.12 & 0.14 & 1.74 & 0.10 \\
\hline 13.082 & 18.21 & 0.04 & 0.70 & 0.06 & 0.05 & 0.06 & & & & & -0.03 & 0.18 & 1.77 & 0.13 \\
\hline 18.7 & 19.02 & 0.08 & 1.11 & 0.21 & 0.06 & 0.14 & 0.84 & 0.15 & 0.55 & 0.16 & -0.11 & 0.17 & 1.34 & 0.14 \\
\hline 21.1 & 19.03 & 0.09 & 1.05 & 0.12 & 0.17 & 0.15 & 1.16 & 0.25 & -0.21 & 0.25 & -0.08 & 0.18 & 1.04 & 0.16 \\
\hline 26.0 & 19.60 & 0.17 & 1.24 & 0.32 & -0.01 & 0.23 & & & & & & & & \\
\hline 31.7 & 19.57 & 0.10 & 0.93 & 0.14 & 0.42 & 0.26 & & & & & & & & \\
\hline
\end{tabular}

Notes. The quantity $t-T_{0}$ is given in days in the observer frame. The data in this table are not corrected for the reddening and light contribution of the host galaxy.

spectral region from the optical to the cosmic ultraviolet. It can obtain an image in only a single filter at any given time. It is therefore necessary to interpolate the observations in the individual filters to determine the color index, especially during the early phase of the OA when the brightness changes rapidly. The color index is calculated as the difference of magnitudes in two, usually neighboring filters. The need to interpolate is strengthened by the fact that the observed data in each light curve are not strictly equidistant. The profile of the light curve in each filter was therefore inspected carefully and found to be wellmapped by the individual observations even in Phase I, with the exception of the gap in $1.5 \mathrm{~d}<t-T_{0}<3 \mathrm{~d}$. It displayed gradual light variations without abrupt changes and fluctuations in the well-mapped phases.

Interpolation of UVOT data to obtain the color indices was done in the following way. In some parts, the variations in the profile of the lightcurve in a given filter were greater than in the remaining filters. In such cases, the time at which the color index was determined was set close to the observed point lying in that curve to avoid distortions. In some cases, splines were used for the interpolation. The standard deviation $\sigma$ usually varied very little from point to point in a given filter in Phase I. It allowed us to attribute $\sigma$ of the surrounding observed points to the interpolated point. For the very late phase mapped by our observations $\left(t-T_{0}>18 \mathrm{~d}\right)$, the scatter of the light curves increased because the $\mathrm{SN}$ faded considerably. The mean magnitude was therefore determined for a group of data in a given filter spanning several days. It was then used to calculate the color index. The brightness faded relatively slowly in this late phase, so this approach is feasible. The resulting color indices and $B$ band magnitudes are listed in Table 1 . The $B$ magnitude is included to show the relation of the color indices to the evolution of the light curve. The standard deviations $\sigma$ of the color indices were calculated from the quoted errors of the data in each filter in the case of a single point or from the standard deviations of the means in the case of a centroid or a bin. The data in this table are not corrected for the reddening and light contribution of the host galaxy, because the magnitudes of the host in the UV band are extrapolations (see below). The corrections of the indices can be applied in the future, if the far UV data of the host become available. The overlapping bands of Mirabal's et al. (2006) data and the $U B V$ UVOT measurements were found to be in good agreement within the errors. A part of the $U$ band light curve was an exception. It is discussed and interpreted below.

For the purpose of our analysis, UVOT data were corrected for the reddening and light contribution of the host galaxy in the following way. The value $E_{B-V}=0.142$ (Mirabal et al. 2006) and the average Galactic extinction curve by Fitzpatrick $\&$ Massa (2007) for $\mathfrak{R}=3.1$ were used to determine $A_{B} / A_{V}=$ 1.32, $A_{U} / A_{V}=1.56, A_{U V W 2} / A_{V}=2.59, A_{U V M 2} / A_{V}=3.19$, and $A_{U V W 1} / A_{V}=2.29$. The $B V R I$ magnitudes of the host galaxy given by Mirabal et al. (2006), Ferrero et al. (2006), and Sollerman et al. (2006) were found to be in good mutual agreement, but discrepancies appeared in the $U$ band. As a plausible approximation, it was decided to make a linear fit to the Sollerman et al. (2006) BVRI magnitudes and to extrapolate it to shorter wavelengths $\lambda$. This enabled us to determine the magnitudes of the host for $U, U V W 1, U V M 2$, and $U V W 2$. For the sake of consistency, the new values of $A_{U} / A_{V}$ and the $U$ band host magnitude we used to recalculate the $U$ band magnitudes in 
Mirabal's et al. (2006) data. It was decided to restrict our analysis to $t-T_{0}<19 \mathrm{~d}$ and $t-T_{0}<26 \mathrm{~d}$ for UVOT and Mirabal's et al. (2006) data, respectively. This represents the interval in which optical emission of the OA is significantly brighter than the host. The uncertainties in the host's magnitude thus do not invalidate the results.

In most cases, the UBVRI data by Mirabal et al. (2006) were obtained during a single night, so calculating the color indices from the magnitudes in the appropriate filters was straightforward. The quantity $\sigma$ of each color index was calculated from $\sigma$ of the magnitude in each appropriate band. The $(V-R)_{0}$ index of the early phase of the OA was determined from the combination of the Mirabal et al. (2006) $R$ band data and the $V$ band UVOT observations. This index was determined from the $R$ band data and an interpolation between two consecutive $V$ band data.

Unless otherwise stated, the color indices of the OA and their $t-T_{0}$ throughout this paper are in the observer's frame to allow a comparison with the papers by Šimon et al. (2001, 2004a,b). For consistency, $t-T_{0}$ of the remaining $\mathrm{SNe}$ plotted are recalculated for $z$ of GRB 060218 .

Time evolution of the color indices of the OA using UVOT data for $0.08 \mathrm{~d}<t-T_{0}<19 \mathrm{~d}$ is displayed in Fig. $1 \mathrm{~b}$. In most cases, the color indices determined from the magnitudes in the neighboring bands were used. In addition, the $(U V W 2-B)_{0}$ index that relates the evolution at two very separated spectral regions was included. To assess the relation between the color indices and magnitude, the $B$ band light curve is displayed in Fig. 1a. These data map the rising branch, the peak, and the initial part of the decay of the first bump in the light curve in Fig. 1a. Only small color changes occurred in $0.08 \mathrm{~d}<t-T_{0}<0.5 \mathrm{~d}$, i.e. on the rising branch and the broad peak. The gap in the coverage in $1.5 \mathrm{~d}<t-T_{0}<3 \mathrm{~d}$ makes the assessment of the color evolution during the transition between Phase I and the emerging SN uncertain, but some trends are evident. The separation of the colors appropriate for Phase I and for SN 2006aj is clear (with the decreasing amplitude) for $(U V W 2-B)_{0},(U V W 1-U)_{0}$, and $(U V M 2-U V W 1)_{0}$. Especially $(U V W 1-U)_{0}$ and $(U V M 2-U V W 1)_{0}$ only display small variations during Phase I. Their evolution during Phase II (the SN phase) is almost achromatic, too, but at values quite different from those appropriate to Phase I. The situation is very different for $(U V W 2-U V M 2)_{0}$, which displays almost identical mean values for both phases.

The light curves of the optical emission in the individual bands, focused on the evolution of SN 2006aj, are displayed in Fig. 2. The times of the peak light, $T_{\max }$, in the $U B V R I$ filters considerably differ for the individual bands, and the decay rate increases with the decreasing $\lambda$. When going to even shorter $\lambda$, i.e. to $U V W 1, U V M 2$, and $U V W 2$, the situation becomes more complicated. The reason is that the slope of the decaying branch does not follow the trend seen between $U$ and $I$. The decay rate in $U V W 1$ is comparable to the one in $B$. The decaying branches in $U V M 2$ and $U V W 2$ are mutually similar and clearly differ from those at longer $\lambda$. The peak brightness cannot be resolved with certainty in these two bands. Instead, these light curves display a plateau or at most a slow decline until $t-T_{0} \approx 13 \mathrm{~d}$. Only in the final phase can the decay rate be comparable to rate in the $B$ band. In $U V M 2$, the time of the peak light that is almost coincident with that in the $U$ band can be barely visible.

It was decided not to try to separate the light curve of Phase I from the one in SN 2006aj. The reasons follow. Only the first part of the decline of Phase I (i.e. after the first peak light in the $B$ light curve) is mapped in Fig. $1 \mathrm{~b}$, so the real profile of the second part of the decline cannot be determined with certainty.

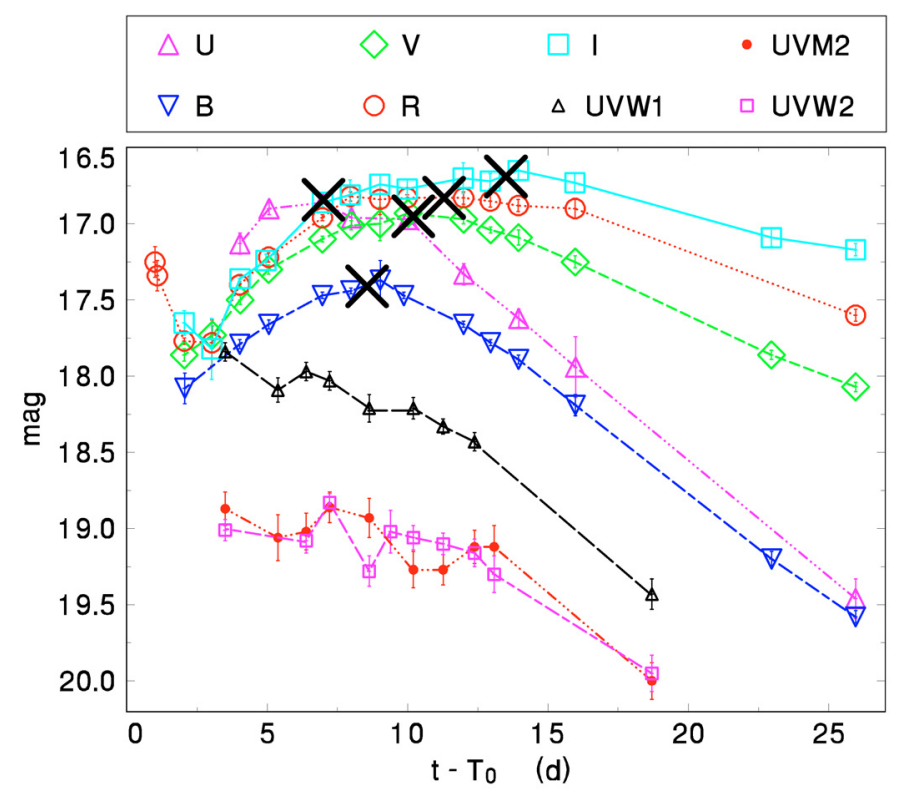

Fig. 2. The light curves of the OA of GRB 060218 in the individual bands. The time interval $t-T_{0}$ is in the observer frame. This diagram concentrates on the evolution of SN 2006aj, so most data from Phase I are skipped to avoid overcrowding. The data are corrected for the Galactic extinction and contribution of the host galaxy. The UBVRI data come from Mirabal et al. (2006), the rest obtained with UVOT. The points are connected by lines for convenience. The large crosses denote the time of the peak light in a given filter (for $U B V R I$ ). See Sect. 3 for details. (This figure is available in color in electronic form.)

This would make the separation highly speculative. Moreover, it is not known with certainty where the light of Phase I comes from, i.e. whether it originates in the jet or in a part of the outer layer of the SN. It is thus not known how much this procedure is justified from the physical point of view (see below).

Before studying the color indices, we investigated how the time of the rest-frame peak light of SNe, $\left(T_{\max }-T_{0}\right)_{\text {rest }}$, depends on the rest-frame wavelength $\lambda_{\text {rest }}$. These variations were studied for three hypernovae with a dense coverage by the multiband photometric data: SN 2006aj, SN 1998bw (data of Galama et al. 1998), and SN 2002ap (data of Yoshii et al. 2003). The time of the peak light in rest frame, $T_{\text {max }_{\text {rest }}}$, that is measurable in the light curves for the individual bands can provide us with information on the evolution of the SN outer layers. For any given $\mathrm{SN}$, we determined the time of the peak light $T_{\text {max }}$ in the light curve in each band. The effective wavelength of each filter, $\lambda_{\mathrm{eff}}$, was used for our purpose. Since all SNe used were in the photospheric phase where most light comes from the continuum, this approximation is feasible. We then determined the values of $T_{\max }-T_{0}$. The quantities $\lambda_{\mathrm{eff}}$ and $T_{\max }-T_{0}$ were transformed to the rest-frame values $\lambda_{\text {rest }}$ and $\left(t-T_{0}\right)_{\text {rest }}$ according to $z$ of the given SN (SN 2006aj and SN 1998bw lie at cosmological distances). Only SN 2002ap is nearby, at $z \approx 0$, since it is located in M 74 (Nakano et al. 2002). The time $T_{0}$ in JD 2450929.41 (set by the associated GRB 980425) (Soffitta et al. 1998) and $z=0.0085$ (Tinney et al. 1998) were used for SN 1998bw. Since $T_{0}$ is not accurately known for SN 2002ap, $T_{\max }-T_{0}=10 \mathrm{~d}$ was assumed for the $V$ band (Mazzali et al. 2002). For SN 2006aj, the time of the peak light in the $U V W 1$, $U V M 2$, and $U V W 2$ bands could not be determined, because this peak is not defined very well in Fig. 2. The light curve displays a broad plateau instead of any measurable peak. The result is displayed in Fig. 3. The quantity $\left(T_{\max }-T_{0}\right)_{\text {rest }}$ clearly varies 


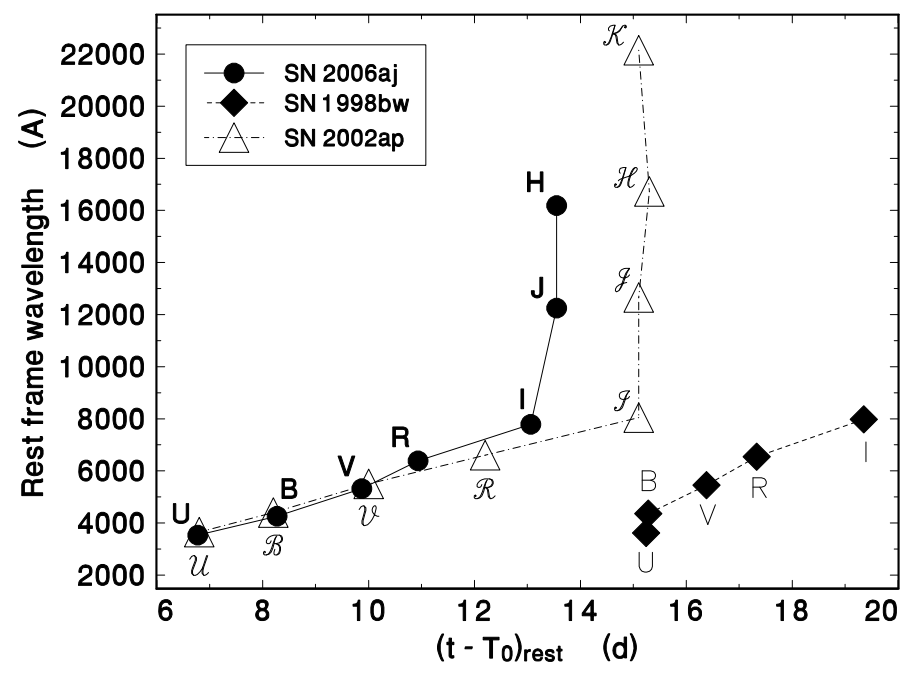

Fig. 3. The time of the peak light of SN vs. wavelength. Both the effective wavelength and the time interval $\left(t-T_{0}\right)_{\text {rest }}$ are expressed in the rest frame. The filter used is marked for each data point. Three type Ic hypernovae with a sufficient coverage by the data are displayed. The points are connected by lines for convenience. See Sect. 3 for details.

with $\lambda_{\text {rest }}$ for a large part of the spectrum of a given SN used in Fig. 3. Only the observation of SN 2006aj in $J$ and $H$ and of SN 2002ap in $J H K_{\mathrm{s}}$ deviate. Indeed, a prominent and abrupt change in the $\left(T_{\max }-T_{0}\right)_{\text {rest }}$ vs. $\lambda_{\text {rest }}$ relation occurs here. We call this change at $\lambda_{\text {rest }} \approx 7000 \AA$ the stagnation point, beyond which the time of the peak light remains independent of $\lambda_{\text {rest }}$. Although the slope of the relation shortward of the stagnation point is almost identical for SN 2006aj and SN 2002ap, their stagnation points differ. The $J H K_{\mathrm{S}}$ data are unavailable for SN 1998bw, but its stagnation point (if any) definitely occurs later $\left(t-T_{0}\right)_{\text {rest }}$ than with SN 2006aj and SN 2002ap. We quantify the progress of $\left(T_{\max }-T_{0}\right)_{\text {rest }}$ with $\lambda_{\text {rest }}$ shortward of the stagnation point in terms of the shift of $\lambda_{\text {rest }}$ per day. We thus obtain the shift of $690 \AA \mathrm{d}^{-1}$ for SN 2006aj, $550 \AA \mathrm{d}^{-1}$ for SN 2002ap, and $1000 \AA \mathrm{d}^{-1}$ for SN 1998bw.

The time evolution of the color indices of the OA of GRB 060218 between the $B$ and $I$ bands is displayed in Fig. 4. To show the evolution of SN 2006aj in more detail, only the interval of $t-T_{0}$ between 0.8 and 50 days is displayed. To place this event in the context, we also show the evolution of SNe: SN 1998bw, SN 2002ap, the groups of type Ib and type Ic SNe from the database of Poznanski et al. (2002).

We generated synthetic color indices of SN 1998bw, SN 2002ap, and type Ib and Ic SNe using the code at http://wise-obs.tau.ac.il/ dovip/typing (Poznanski et al. 2002), with both $\lambda$ of the bands and $t-T_{0}$ scaled to $z=0.03345$. The quantity $T_{0}$ refers to the time of GRB 980425 for SN 1998bw. The peak magnitude of SN 1998bw occurred at $t-T_{0} \approx 16.5 \mathrm{~d}$ for $z=0.03345$. We also generated the colors of SN 2002ap and the group of types Ib and Ic SNe in the same way. In these cases, the time scales were recalculated by taking the rise time to the maximum brightness $\sim 10 \mathrm{~d}$ at $z \approx 0$ (Mazzali et al. 2002). Any small uncertainty in the rise-time scale will not influence our results. Those indices inside a similar interval of $t-T_{0}$ to the one mapped for the OA of GRB 060218 are included in Figs. 4 and 5. Since these synthetic spectra come from the ground-based measurements, they could not be applied to the observations in the $U V W 1, U V M 2$, and $U V W 2$ bands. Except type $\mathrm{Ib}$, the color indices of all other types of SN displayed in Fig. 4 initially rise, but the times of their maximum and their peak values differ. The largest differences between these indices occur right on this rising branch and allow us the most reliable separation of the individual types. Generally, the maximum of the color index does not coincide with the peak brightness of that SN type.

The time evolution of SN 2006aj in Fig. 4 differs from those of all other core-collapse $\mathrm{SNe}$ displayed. We attempted to investigate whether stretching the time evolution of the $\mathrm{SN}$ can help solve this problem. After several trials, it was found that a stretching of the time evolution of SN 1998bw by a factor of 0.5 (i.e. SN 1998bw evolving twice as fast) yields plausible fits in both $(B-V)_{0}$ and $(V-R)_{0}$ between these $\mathrm{SNe}$. As for $(R-I)_{0}$, SN 2006aj was systematically redder and its color consistent with that of SN 2002ap and the ordinary type Ic. Stretching the time evolution of the group of type Ic SNe by a factor of 1.2 yielded a plausible fit to the time evolution of $(B-V)_{0},(V-R)_{0}$, and $(R-I)_{0}$. A comparison with fitting the light curves by other authors shows that Ferrero et al. (2006) determined the stretch factor of 0.62 in $B$, while it was 0.68 in $V R I$, taking SN 1998bw as the template. Valenti et al. (2008) conclude that the stretch factor is 0.73 for the $V$ band light curve with respect to SN $1998 \mathrm{bw}$. The time evolution of $(U-B)_{0}$ is not shown in Fig. 4. The value of this index increases with time but is lower than that of all other SNe used in Fig. 4 for a given $t-T_{0}$. It therefore cannot be matched by their color index. In summary, after appropriate stretching, type Ic matches the color evolution of SN 2006aj over a broader spectral range than that of the "canonical" SN 1998bw.

The color indices were also plotted in the color-color diagrams (Figs. 5 to 9) to allow investigation of their shifts in more detail. Since the profiles of the color evolutions in some colorcolor diagrams were mutually similar, their number was reduced. The $(V-R)_{0}$ vs. $(R-I)_{0}$ diagram is similar to the $(B-V)_{0}$ vs. $(V-R)_{0}$ diagram, so only the latter is displayed in Fig. 5. As shown below, only the color evolution of SN 1998bw and type Ic $\mathrm{SNe}$ were found to be able to yield plausible matches to SN 2006aj. The other SNe are therefore not shown to avoid overcrowding in the color-color diagrams.

We find differences in the value of $(U-B)_{0}$ between the data from UVOT and Mirabal et al. (2006, Fig. 6). The slight differences between Johnson $U$ and UVOT $U$ (Poole et al. 2008) cannot be the sole explanation because these discrepancies only occur in some phases of SN 2006aj. We ascribe them to the role of the steeply falling flux between the $U$ and $B$ band of the SN spectrum with time. Even a small difference in the filters used in the different telescopes can then create this effect.

For comparison, the mean colors with the standard deviation of the ensemble of OAs of long GRBs $\left(t-T_{0}<10 \mathrm{~d}\right.$ ) (Šimon et al. 2004a) are also shown in Figs. 4 and 5. We note that our previous analysis (Šimon et al. 2001, 2004a) was restricted to $t-T_{0}<10 \mathrm{~d}$ for practical reasons, since for most OAs the data for later epochs are sparse, with large uncertainties, and mostly only in the $R$ band. The colors of these OAs are found to be independent of redshift for $z=0.43-3.5$ (see Šimon et al. 2001, for details). The clustering of their color indices is caused by one observe a part of the synchrotron spectrum of the OAs with a very similar slope in the individual events and by the absence of dust in the line of sight in their host galaxies (Šimon et al. 2006). Although the color indices of this ensemble were determined for $z=0.43-3.5$, we argue that it is possible to use them for an analysis of the event at $z=0.03345$, too, because the $(I-$ $J)_{0}$ index in Šimon et al. (2004a) suggests that the slope of the 

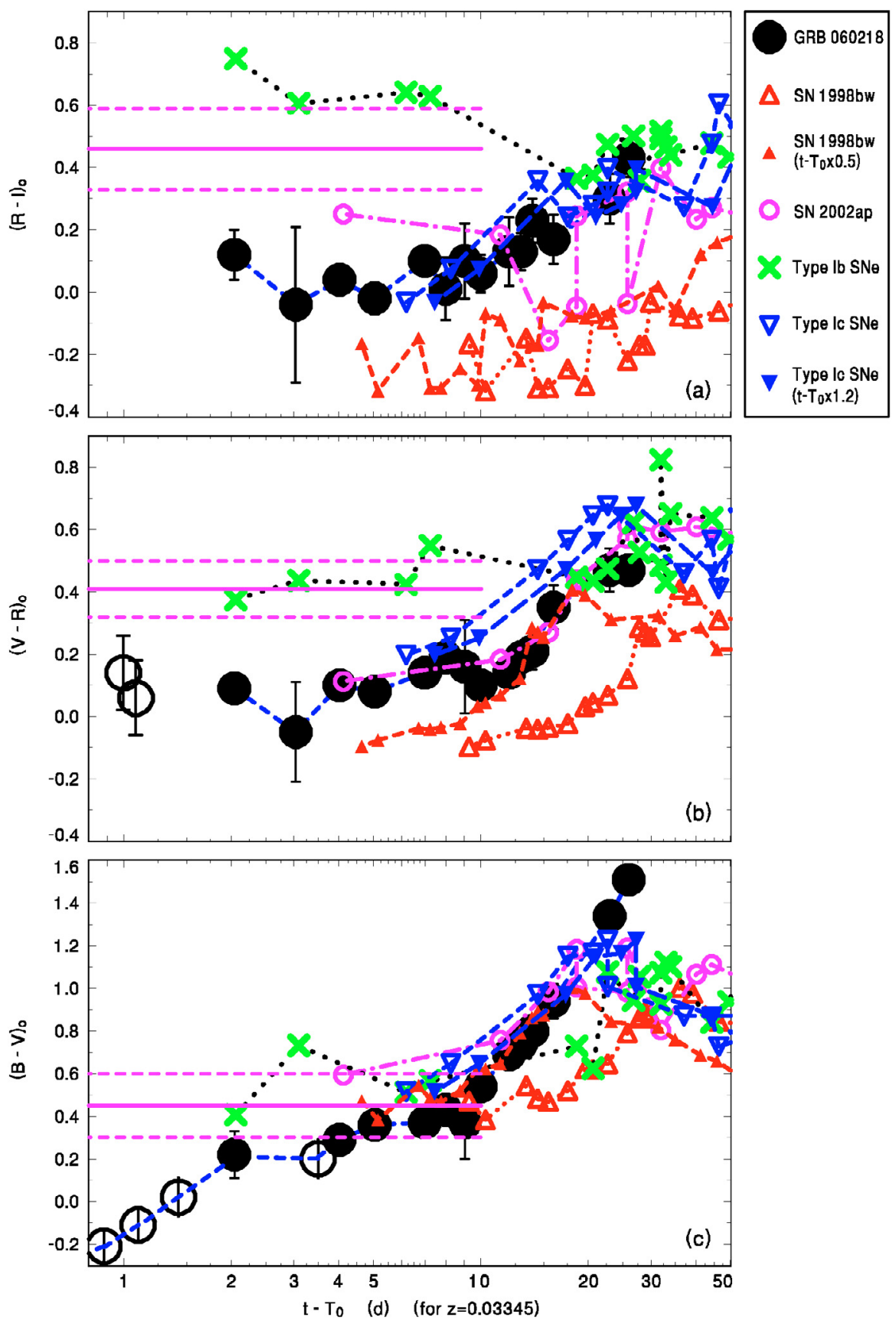

Fig. 4. Time evolution of the color indices of the OA of GRB 060218. Large solid and open circles denote the data by Mirabal et al. (2006) and from UVOT, respectively. The points are connected by lines for convenience. The horizontal solid line with the dashed error bars marks the mean color indices of the ensemble of OAs, determined by Šimon et al. (2004a). The synthetic colors of SN 1998bw, SN 2002ap, the group of type $\mathrm{Ib} \mathrm{SNe}$, and the group type Ic SNe (Poznanski et al. 2002), with the bands and $t-T_{0}$ recalculated for $z=0.03345$, appropriate for GRB 060218, are also plotted. The colors of OAs are found to be independent of the redshift. See Sect. 3 for details. The time stretch factor, which is discussed in Sect. 4.3, is not included. (This figure is available in color in electronic form.)

synchrotron spectrum continues for long enough $\lambda$ to allow a meaningful comparison with Phase I of the OA of GRB 060218.

Figures 4 and 5 show that the color indices of the OA of GRB 060218 for Phase I differ from those of the ensemble of OAs. The $(B-V)_{0}$ and $(V-R)_{0}$ indices overlap with the data for which the ensemble was made. This enables a meaningful comparison. The $(U-B)_{0}$ index of the ensemble of OAs displayed a considerable scatter due to the change of the spectral profile of some OAs (Šimon et al. 2006). The grouping of the color indices is clearly visible in Phase I of the OA of GRB 060218, too, although at different values from those of the above-mentioned ensemble. In the case of GRB 060218, this grouping also considers the cosmic UV band. The mean color indices for $0.08 \mathrm{~d}<$ $t-T_{0}<0.55 \mathrm{~d}$ were determined as $(B-V)_{0}=-0.15 \pm 0.10$, $(U-B)_{0}=-1.44 \pm 0.03,(U V W 1-U)_{0}=-0.73 \pm 0.04$, $(U V M 2-U V W 1)_{0}=-0.54 \pm 0.03,(U V W 2-U V M 2)_{0}=$ $0.06 \pm 0.07$, and $(U V W 2-B)_{0}=-2.66 \pm 0.05$.
It was also decided to include the color-color diagram that relates the evolution in two very separated spectral regions (Fig. 7). To be consistent with the data products of the code by Poznanski et al. (2002), we determined the sum of the indices $(U-B)_{0}$ and $(B-V)_{0}$ for the observed data of SN 2006aj instead of calculating $(U-V)_{0}$. For the same reason, the $(V-R)_{0}+(R-I)_{0}$ index was determined.

A large diagonal shift of SN 2006aj is the common feature in the color-color diagrams in Figs. 5-7. On the other hand, the shift of SN 1998bw displays a strikingly small amplitude. The remarkable feature in these color-color diagrams is the so-called turning point, which means that the shift of the SN makes a loop and begins to evolve backward. The turning point is the tip of this loop. This term was introduced by Richmond et al. (1996) for the evolution of the color index of SN versus time. Hereafter we use it for the color-color diagrams. This point occurs in the path of both SN 1998bw and the group of type Ic SNe in Figs. 5-7, 


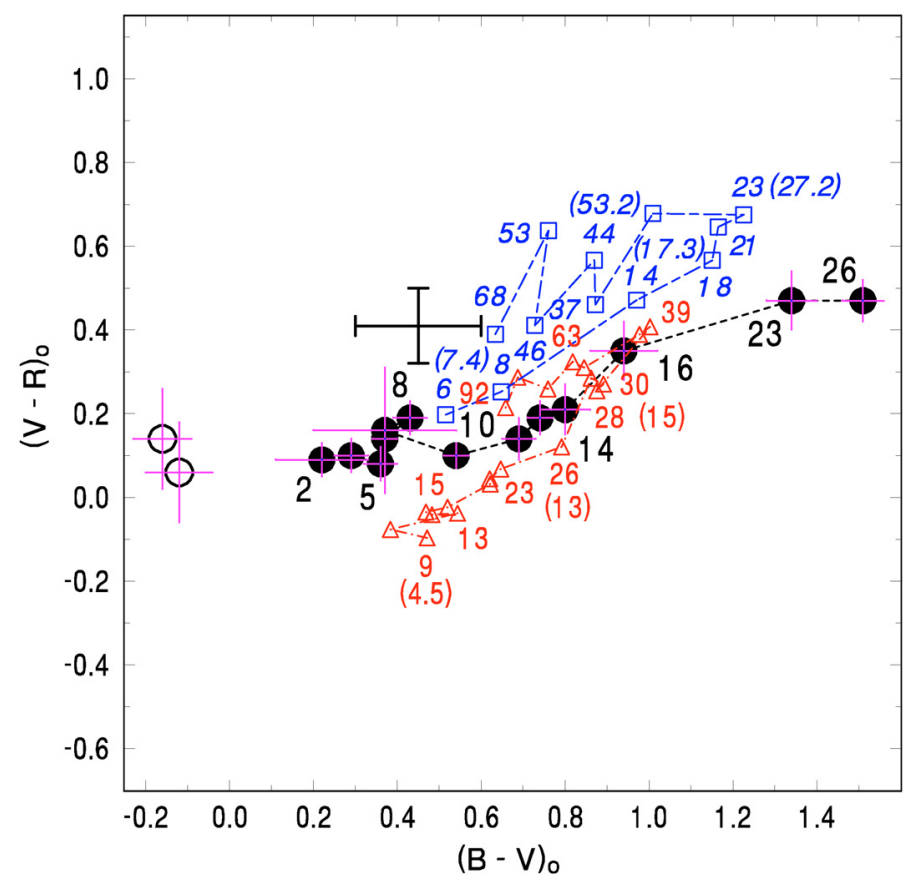

Fig. 5. $(B-V)_{0}$ vs. $(V-R)_{0}$ diagram of the OA of GRB 060218. Solid circles denote the data by Mirabal et al. (2006). Open circles mark the colors determined from the combination of Mirabal's et al. and UVOT data. The points are connected by a line to show the time evolution of the color indices. The large cross denotes the centroid and standard deviations of the colors of the ensemble of OAs for $t-T_{0}<10 \mathrm{~d}$ (Šimon et al. 2001, 2004a). Open triangles and open boxes mark the synthetic colors of SN 1998bw and type Ic SNe, respectively. The numbers at the points denote $t-T_{0}$ in days for $z=0.03345$; it starts from the appropriate GRBs in the case of GRB 060218 and SN 1998bw. In the case of type Ic $\mathrm{SNe}, t-T_{0}=0 \mathrm{~d}$ is set to $10 \mathrm{~d}$ before the time of the $V$ band peak light. The numbers in brackets refer to SN 1998bw and type Ic SNe evolving twice as fast and by a factor of 1.2 slower, respectively. See Sect. 3 for details. (This figure is available in color in electronic form.)

but its position differs considerably. The loop is absent from all displayed $\mathrm{SNe}$ in the $(V-R)_{0}$ vs. $(R-I)_{0}$ diagram, which means that it is confined to $\lambda<5300 \AA$ for $z=0.03345$. This loop is not observed in SN 2006aj in Figs. 5-7, possibly because it occurred after the end of the observations of this object. The turning point occurs on the decaying branch of the optical light curve, not at the maximum light, and its position plays a role in the amplitude of the shift in the color-color diagram. The most negative colors occur during the first several days, that is, on the rise to the peak light; this is the common property of all the $\mathrm{SNe}$ in these diagrams until the turning point is reached. The colors then gradually become more positive on the decaying branch of the light curve. SN 2006aj displays the largest shift in Fig. 7; the reddening is mostly due to the variations in the $(U-B)_{0}+$ $(B-V)_{0}$ index, while in the $(V-R)_{0}+(R-I)_{0}$ direction it displays a comparable amplitude to the remaining two cases (i.e. $\mathrm{SN} 1998 \mathrm{bw}$ and the group of type Ic SNe). Only small variations in the color indices occurred on the rising branch of the light curve; SN 2006aj changed by only $\sim 0.25$ mag in $(V-R)_{0}+(R-I)_{0}$ between $t-T_{0}$ of 4 and $10 \mathrm{~d}$. SN 2006aj starts with an initially rapid shift from an extremely negative $(U-B)_{0}+(B-V)_{0}$ index. It reaches the turning point at quite a similar $t-T_{0}$ as type Ic. If we state it more precisely, only type Ic SNe approach this turning point in the "right" value of the color index, although this is just the lower limit in the case of SN 2006aj. SN 2006aj definitely must display the most positive $(U-B)_{0}+(B-V)_{0}$ of

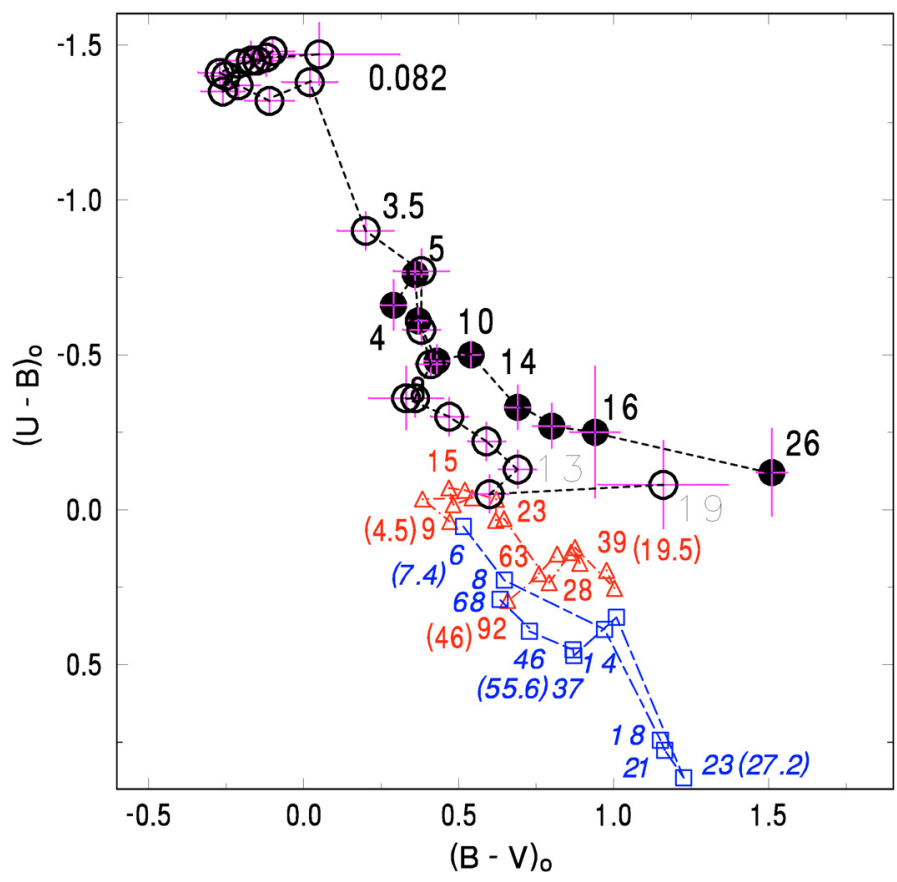

Fig. 6. $(U-B)_{0}$ vs. $(B-V)_{0}$ diagram of the OA of GRB 060218. Open and solid circles denote the data from UVOT and those by Mirabal et al. (2006), respectively. The arrangement is analogous to Fig. 5. (This figure is available in color in electronic form.)

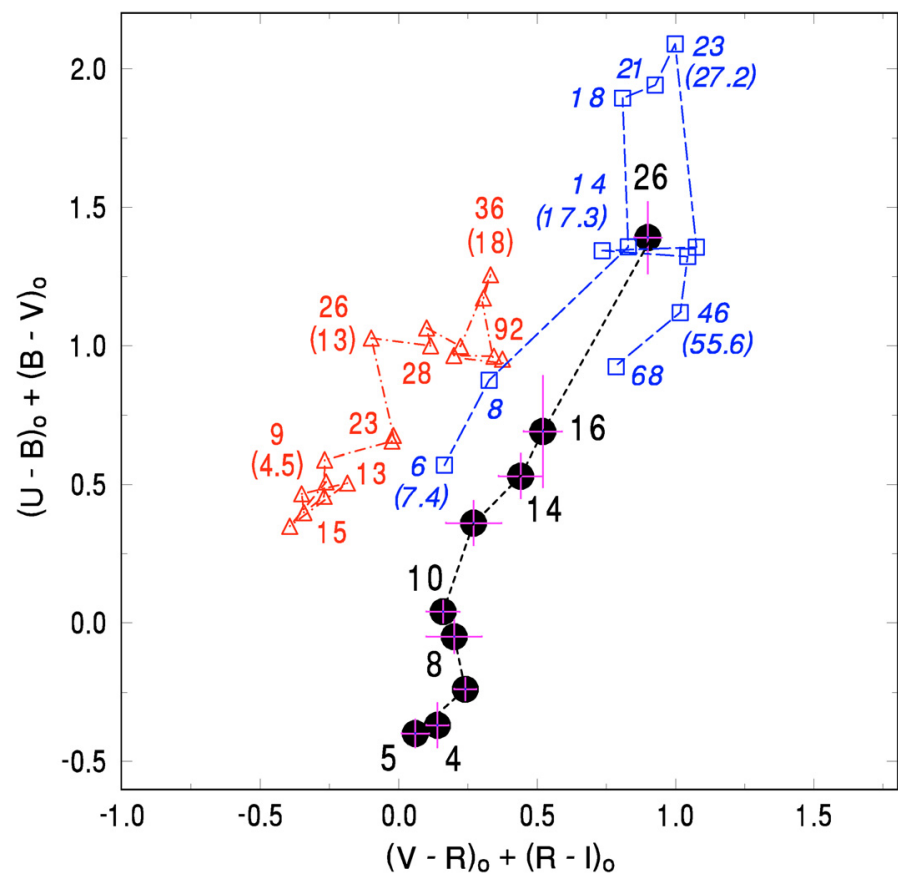

Fig. 7. $\left.(U-B))_{0}+(B-V)\right)_{0}$ vs. $\left.\left.(V-R)\right)_{0}+(R-I)\right)_{0}$ diagram of the OA of GRB 060218. The arrangement is analogous to Fig. 5. See Sect. 3 for details. (This figure is available in color in electronic form.)

the turning point among the plotted SNe. It is much redder than in the canonical SN 1998bw.

The situation in the color-color diagrams of the UV region (Figs. 8 and 9) is quite different from that at longer $\lambda$. The $(U V W 2-U V M 2)_{0}$ vs. $(U V M 2-U V W 1)_{0}$ diagram is similar to the $(U V M 2-U V W 1)_{0}$ vs. $(U V W 1-U)_{0}$ diagram, so only the latter is displayed in Fig. 8. Both of them show two largely separated groups of data. The former belongs to Phase I, 


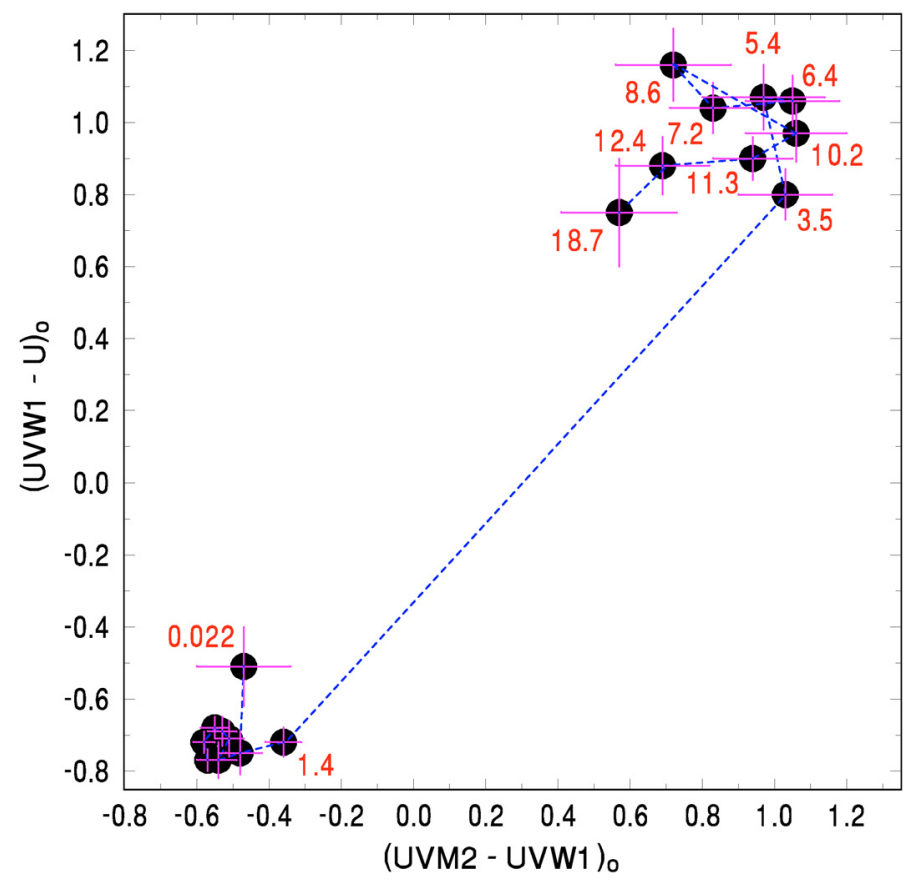

Fig. 8. $(U V M 2-U V W 1)_{0}$ vs. $(U V W 1-U)_{0}$ diagram of the OA of GRB 060218. The UVOT data are used. The points are connected by the line to show the time evolution of the colors. The numbers at the points denote $t-T_{0}$ in days. The data were corrected for the reddening and light contribution of the host galaxy. (This figure is available in color in electronic form.)

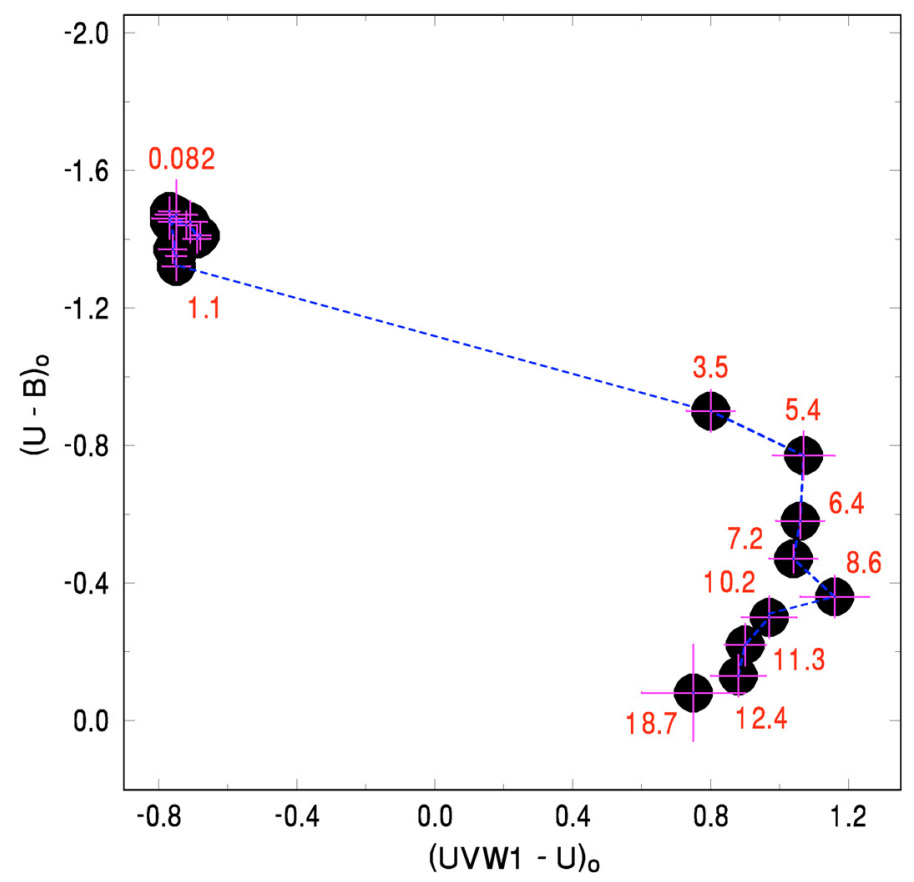

Fig. 9. $(U V W 1-U)_{0}$ vs. $(U-B)_{0}$ diagram of the OA of GRB 060218. The arrangement is analogous to Fig. 8. The data were corrected for the reddening and light contribution of the host galaxy. (This figure is available in color in electronic form.)

the latter contains the data of Phase II, that is, SN 2006aj. Also the points that belong to Phase II display only small time evolutions in these diagrams. Figure 9 represents the time evolution of the OA in a very important spectral region. While a prominent clustering occurs in Phase I, a large evolution of both
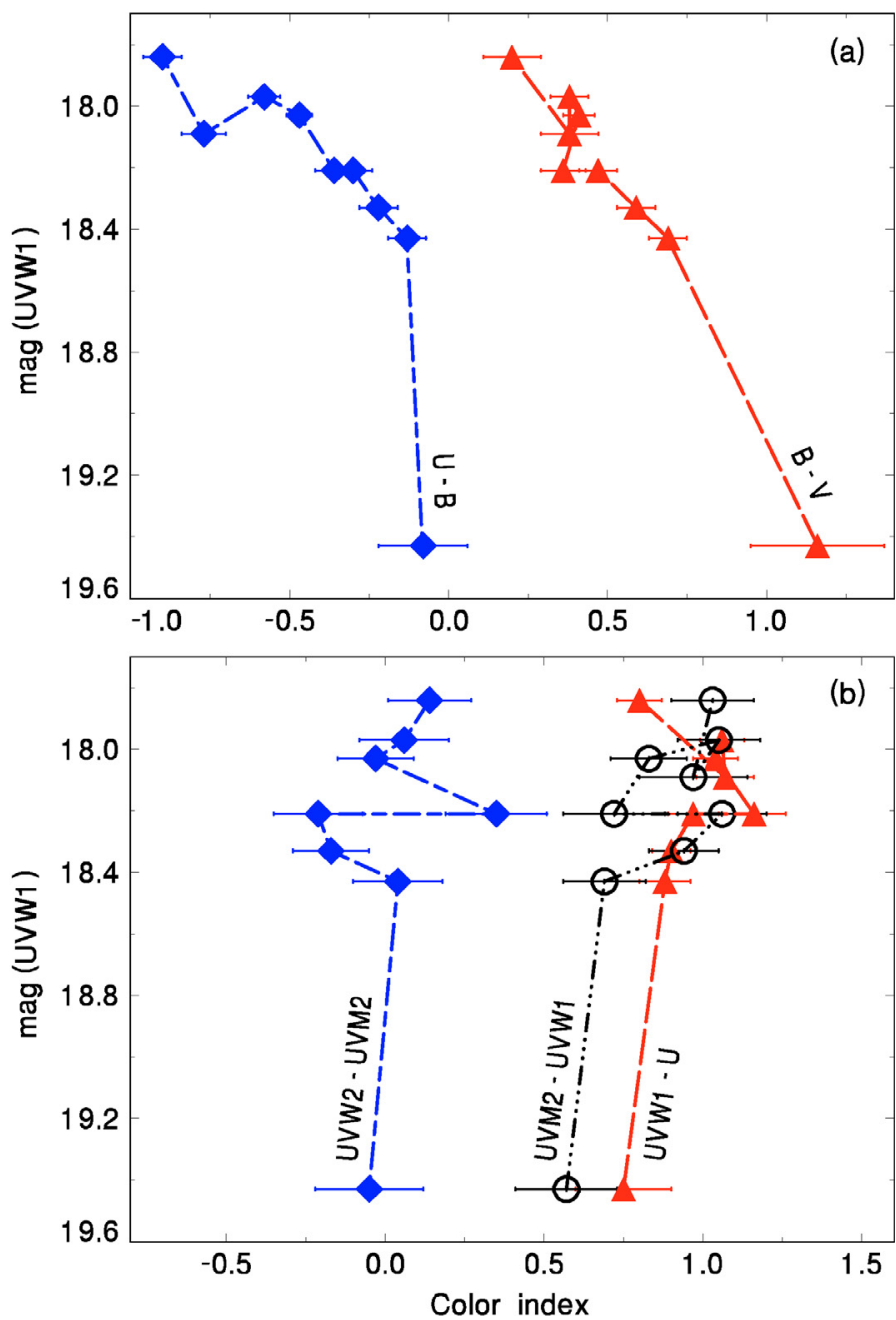

Fig. 10. The color index vs. magnitude diagram for SN 2006aj (3.5 d < $t-T_{0}<18.7 \mathrm{~d}$ ). a) The colors in the band longward of the region, in which line blanketing dominates. b) The colors in the region with line blanketing. Error bars of the color index are included. The data were corrected for the reddening and light contribution of the host galaxy. See Sect. 3 for details. (This figure is available in color in electronic form.)

$(U V W 1-U)_{0}$ and $(U-B)_{0}$ indices is observed in Phase II. Also the $(U V W 2-U V M 2)_{0}$ vs. $(B-V)_{0}$ diagram displays a clustering of the data only in Phase I, while a gradual increase in $(B-V)_{0}$ followed through Phase II. A transient maximum of $(U V W 2-U V M 2)_{0}$ was observed at $t-T_{0}=1.4 \mathrm{~d}$, which is near the minimum of the $B$ band brightness separating Phase I from Phase II.

The evolution of the color indices with the magnitude of SN 2006aj ( $\left.3.5 \mathrm{~d}<t-T_{0}<18.7 \mathrm{~d}\right)$ is displayed in Fig. 10, showing the different shifts, when the diagrams in Figs. 10a and $\mathrm{b}$ are compared.

\section{Discussion}

We present the results of our analysis of the photometric properties of the OA of GRB 060218. Our approach, which makes use of the color indices, traces the time evolution both of the early afterglow (Phase I) and of the SN outer layers (Phase II) in various spectral regions including the cosmic UV band $(\lambda>188 \AA)$. We show that it provides us with more pieces of information on the processes than the light curves can do themselves. 


\subsection{Phase I of the $O A$}

Although the color indices of Phase I suggest the spectral profile slightly different from that of the ensemble of OAs of long GRBs that we analyzed before (Šimon et al. 2001, 2004a), they share the tendency to cluster in the color-color diagrams and to only display small variations in time. Especially the initial clustering of the color indices in the cosmic UV band is very similar to the behavior of the colors of the ensemble of OAs that we determined for longer $\lambda$. We find a very large difference between the values and time evolution of the color indices of the OA of GRB 060218 when we compare Phases I and II. This suggests that we observed two different phenomena before and after this time. We show in detail in Sect. 4.4 that the emission of the initial phase occurred significantly higher above the photosphere than the subsequent SN light. We interpret the large change of $U V W 2-B$ as the transition between these two light-emitting components. The large shift in $U V W 2-B$ started already at $t-T_{0} \approx 0.55 \mathrm{~d}$, which is shortly after the peak of the first bump in the light curve in Fig. 1a. We interpret it as the increasing light contribution of the outer layers of SN 2006a. Most color indices of Phase I differ significantly from those of the subsequent SN 2006aj (Fig. 1). Since the $U V W 2-B$ index relates the evolution in two distant spectral regions, the time evolution of these two components can be distinguished in detail. It thus emerges that the emission of Phase I influenced the light of the subsequent SN 2006aj at most marginally (Figs. 8 and 9). Phase I of this OA will be analyzed in detail in a separate paper (Šimon et al. 2010, in prep.).

Even in Phase I of the OA of GRB 060218, we detected no variations in the intrinsic reddening inside its host galaxy. The color indices are very sensitive to a variable reddening, especially in the blue and UV spectral regions. Such changes would be clearly distinguishable in the color-color diagrams for the cosmic UV band. The absence of these variations can be explained as due to the properties of the host galaxy: low dust abundance and very low metal abundance (see Wiersema et al. 2007). Although the density and dust abundance of the interstellar medium local to the GRB (e.g. the dust produced by the progenitor) can be substantially reduced by the intense initial optical-UV flash (see models by Waxman \& Draine 2000), this scenario is unlikely in the case of GRB 060218 for the following reason. UVOT started observing the field of GRB 060218 at $1875 \mathrm{~s}$ after $T_{0}$. It caught the OA's faint and only slowly brightening (Fig. 1a). This puts constraints on the time interval during which any hypothetical, initially strong, narrow spike destroying the dust could occur without being detected by UVOT. Changes in the color indices mainly in the cosmic UV band are also expected if the emitting region is immersed in the stellar wind of the progenitor and gradually emerges from it. Since this progenitor was a Wolf-Rayet star with a strong and dense wind (e.g. Blustin 2007; Sonbas et al. 2008), such a shift in the color indices would be expected if the emitting region were located deep in the photosphere of this star. The observations therefore show that the light of Phase I comes from far above the photosphere and the wind.

\subsection{Evolution of SN 2006aj in various bands}

We present several lines of evidence that show a rapid, pronounced and complicated evolution of the outer layers of SN 2006aj. This is suggested by the spectral profile variation over a broad range of $\lambda$, from the cosmic UV to the $I$ band during
Phase II: (a) shift in the time of the peak light for the individual bands (Fig. 3), (b) time evolution of the color indices (Figs. 48). Changes in a large part of the UV and optical continuum of SN 2006aj, not only of the line(s), must be involved. The reason is that otherwise we would not observe the mutually similar shifts in the individual color-color diagrams.

Tracing this evolution using photometric filters is justified because SN 2006aj was in the photospheric phase during our observations. The spectra taken by Sollerman et al. (2006) during $t-T_{0}<12.3 \mathrm{~d}$ showed the continuum-like spectrum with broad bumps. It is reasonable to suggest that this phase was representative of all our observations. For example, SN 1997ef did not enter nebular phase before more than two months after the start of its explosion (Foley et al. 2003).

The models of the $\mathrm{SN}$ evolution made for the $U$ to $I$ spectral region predict that the peak of the SN light curve is sharper and the decline steeper for shorter $\lambda$ (Woosley et al. 1999). We show that both these properties are fulfilled for type Ic SN 2006aj only between $U$ and $I$, while discrepancies occur for shorter $\lambda$. We can use their model light curves for SN 2006aj since $\lambda$ and $\lambda_{\text {rest }}$ are similar to each other because of the small $z$. The time of its observed peak light follows this predicted trend for $\lambda_{\text {rest }} \geq 3500 \AA$ but it deviates from it for shorter $\lambda_{\text {rest }}$. We show that the decay rate in $U V W 1, U V M 2$, and $U V W 2$ is not faster than in $U$ in the case of SN 2006aj. The observed variation of $\left(T_{\max }-T_{0}\right)_{\text {rest }}$ with $\lambda$ reflects the evolution of the outer layers of $\mathrm{SNe}$ in various spectral regions (Fig. 3). We find the common features in the behavior of three hypernovae: SN 2006aj, SN 1998bw, SN 2002ap. The first two of them are associated with long GRBs. In SN 2006aj, the approximately linear shift of $\lambda$ per day can be traced for $\lambda_{\text {rest }} \approx 3500-8000 \AA$. At shorter $\lambda$, down to $1820 \AA$, only a broad plateau and a decline were observed. The brightness at this $\lambda_{\text {rest }}$ was already decreasing in the time that the brightness between $U$ and $I$ was still rising. Although the slope of the shift between $\lambda_{\text {rest }} \approx 6700$ and $8000 \AA$ is almost identical for SN 2006aj and SN 2002ap, their stagnation points differ from each other. This point definitely occurs at later $\left(t-T_{0}\right)_{\text {rest }}$ for SN $1998 \mathrm{bw}$, whose progenitor was the most massive star in this ensemble. We show that this behavior can be quite similar for the $\mathrm{SNe}$ with a different evolution of the color indices as shown on SN 2006aj and SN 2002ap. A grid of the future detailed models with varying parameters like the mass of the progenitor immediately before the $\mathrm{SN}$ explosion, opacity of the SN, asymmetry of the ejecta, viewing angle is desirable for disentangling the role of the individual parameters in the observed light and spectral evolution.

We find an extremely large time variation of the color indices of SN 2006aj between the $U$ and $I$ bands. They accompany the rapid evolution of its light curve. The $(R-I)_{0}$ and $(V-R)_{0}$ indices provide us with the most reliable discrimination among the individual SN types in our case (Fig. 4). They can thus serve as a good diagnostic tool for the OAs at low $z$. The shifts in the color-color diagrams of SN 2006aj show a different spectral evolution in the parts lying toward the longer and shorter wavelengths from the of $U$ band (Fig. 10). This suggests that we simultaneously observed two spectral regions that were differently evolving. This difference increased with time. The absence of the turning point in the color-color diagrams for the spectral region spanning from $U$ to $I$ can be explained if the outer layers of SN 2006aj evolved so rapidly that its brightness faded below the detection limit before this point could be reached. This point could be reached several days after the group of type Ic SNe but it cannot be matched by SN 1998bw (Fig. 7). 
Our findings are worth discussing in the framework of the asymmetric explosion modeled by Maeda et al. (2006). It explains for SN 1998bw that the steep rise of its luminosity was due to asymmetric explosion, hence to the highly asymmetric outer layers. They became more spherical only after the time of the peak magnitude. This asymmetric explosion is also advocated by Maeda et al. (2007) for SN 2006aj. They argue that the asymmetry might be produced by a jetlike explosion with the jet wider than in SN 1998bw. Gorosabel et al. (2006) explain the reported polarization measurements by the evolution of an asymmetric SN expansion. According to the observations of Maund et al. (2007), the polarization associated with spectral lines implies significant asymmetries of O I $7774 \AA$ and Fe II with respect to each other and to the line of sight.

The large and gradual reddening of the color indices of SN 2006aj between $B$ and $I$ with time can be for several reasons that can even act simultaneously. As noted by Maeda et al. (2006), a less massive progenitor possesses more extended outer layers. According to the model of Arnett (1982), the more massive the ejecta of type I SNe and the less their kinetic energy, the more difficult it is for photons to escape. In this scenario, the photons rapidly escape from the ejecta of the low-mass SN 2006aj. The effective temperature of the observed part of the outer layers thus decreases rapidly. This leads to the rapid and strong reddening of the color indices of SN 2006aj between the $B$ and $I$ bands in Fig. 4. This represents the opposite extreme to the massive SN 1998bw with its small shifts in the colorcolor diagrams. Also an additional process can play a role in SN 2006aj. A reduction of radioactive heating leads to a redder color, as modeled by Iwamoto et al. (1994). Non-uniform, stratified distribution of ${ }^{56} \mathrm{Ni}$ can account for early termination of the heating of some parts of the ejecta. This can contribute to the evolution to the red colors within less than 30 days after $T_{0}$.

The color evolution of SN 2006aj in the blue spectral region displays a striking peculiarity. It started with a significant excess light in the $U$ band with respect to the canonical SN 1998bw. This excess cannot be caused by a light deficiency in the $B$ band, because the initially very negative $(U-B)_{0}$ is not accompanied by $(B-V)_{0}$ redder than in SN 1998 bw. In fact, this evolution was not accompanied by any conspicuously blue indices longward of $B$. In this context we note that we also found a very similar behavior of the color indices in SN 2003dh associated with GRB 030329. The values of these indices are discordant with other type Ic SNe, which are not known to be associated with GRBs. We also found that it appears to play a role in explaining its association with the GRB (Šimon et al. 2004b). SN 2006aj conforms to this scenario.

\subsection{Stretch factor in the color indices}

We find that the very rapid time evolution of the color indices of SN 2006aj cannot be matched by any evolution of type Ib, type Ic SN, or hypernova as they stand, that is, without stretching (Fig. 4). Previous analyzes made use of stretching the light curves, of the individual SNe to match their time evolution to a template. Sometimes, the light curves in more than a single filter were used (Ferrero et al. 2006). Here we use the color indices instead of the light curves for the stretching. This approach enables us not only to investigate the evolution of the outer layers in more detail for a single $\mathrm{SN}$, but also to pick out the differences between the individual objects. We find that stretching the time evolution of the color indices of SN 1998bw (the "canonical" case of the SN-GRB connection) by a factor of 0.5 , i.e.
SN 2006aj evolving twice as fast as SN 1998bw, only yields plausible matches between these two $\mathrm{SNe}$ over the $B$ to $R$ bands. Farther into the red region, between $R$ and $I$, SN 2006aj systematically displayed an excess light, at least within $3 \mathrm{~d}<t-T_{0}<$ $26 \mathrm{~d}$. Its color was consistent with that of SN 2002ap and the "ordinary" type Ic. Stretching the time evolution of the group of the "ordinary" type Ic SNe by a factor of 1.2 yields a plausible fit to the time evolution over a broader spectral region (from $B$ to $I$ ). The adjustment of the time evolution of various color indices thus proves helpful in searching for the proper SN type and for identification of the spectral properties whose evolution deviates from the template. The match of the color indices of SN 2006aj by stretching implies that the outer layers evolve faster, but their spectral energy distribution between $B$ and $R$ remains quite similar to that of SN 1998bw. The strikingly small amplitude of the shift of SN 1998bw in the color-color diagrams can be caused by the high mass of the outer layers of this SN. This especially suggests that the outer layers of SN 2006aj are of a considerably lower mass than those in SN 1998 bw.

Now let us compare the results of our approach with those obtained for SN 2006aj by stretching the SN light curves in the individual filters. Valenti et al. (2008) determined the stretch factor of 0.73 with respect to SN 1998 bw for the $V$ band light curve. This value is slightly higher than our result. Ferrero et al. (2006) determined the stretch factor of 0.62 in $B$ while it was 0.68 in $V R I$, taking SN 1998 bw as the template. This shows that the stretch factor of SN 2006aj varies slightly and depends on the spectral band used for fitting.

Although SN 2002ap has a stretch factor of the lightcurve close to that of SN 2006aj, it is considerably fainter (Ferrero et al. 2006; see also Mazzali et al. 2006). In addition, although the stretching of the light curve of SN 2006aj yields a good fit to SN 2002ap (Ferrero et al. 2006), the evolution of the color indices of these two SNe does not yield a good match (especially in Fig. 6). The same stretch factor can be therefore obtained for $\mathrm{SNe}$ with significantly different absolute magnitudes and color indices. Both the duration and absolute magnitude of SN depend on the mass of ${ }^{56} \mathrm{Ni}$ (e.g. Arnett 1979). This dependence also translates into the stretch factor. Both SN 1998bw and SN 2006aj are associated with GRBs in spite of their very discordant stretch factors. We therefore conclude that the mass of ${ }^{56} \mathrm{Ni}$ is not the main factor for producing GRB. This can be reconciled if the inclination of the axis of the explosion in the asymmetric SN is an essential factor.

The method of the color indices can also help distinguish the proper type in other SNe. It can be used simultaneously with the fitting the light curves of the OA by the template SNe with a grid of the stretch factors (GRB 050525A, Della Valle et al. 2006). This approach can thus remove or at least suppress the ambiguity of the suitable SN types.

\subsection{The role of the opacities}

Two clearly separated regions in the UV color-color diagrams suggest that a very rapid and large change in the opacity of the light-emitting region(s) occurred in the OA of GRB 060218 at $t-T_{0} \approx 2 \mathrm{~d}$, i.e. on the border between Phases I and II. This means that the emission of Phase I occurred in different conditions than those of the subsequent SN 2006aj. As discussed in Sect. 4.1, we interpret the small variability of the UV color indices of Phase I as due to the dominant synchrotron emission from the jet which produced the gamma-ray emission. Especially important is the excursion to the very positive $(U V W 2-U V M 2)_{0}$ at $t-T_{0}=1.3 \mathrm{~d}$, which suggests that 
the opacity evolved non-uniformly in time between Phase I and Phase II. The deep minimum of the $B$ magnitude separating Phase I from Phase II (Fig. 1a), inside of which this excursion occurred, is very different from the plateau in the cosmic UV. We interpret this plateau as the extra emission that remained after Phase I. As shown by Brown et al. (2009), the largest difference between the $U V W 1-B$ index of SN 2006aj and type Ib SN 2007Y occurred right in the early phase and significantly diminished later on. This made SN 2006aj so unique because it displayed its additional light-emitting region.

Although the number of type Ib/Ic SNe observed in the cosmic UV band by UVOT is still small (Brown et al. 2009), SN 2006aj displayed the fastest evolution among them. This SN was reddening all the time longward of the $U$ band in Fig. 7, with the greatest progress between the $V$ and $U$ bands. On the other hand, no significant reddening was observed in the cosmic UV. SN 2006aj thus displayed remarkable differences between the color evolution of the short-wavelength spectral regions where the opacity dominates and the long-wavelength ones where it does not (e.g. Karp et al. 1977; Panagia 2003). We confirm this division by comparing the evolution of the colors in two separated regions with and without high opacity (Fig. 10). Starting from the peak $U V W 1$ magnitude, longward of $U$ the spectrum reddens with the decline of the UV brightness, especially before crossing $18.5 \mathrm{mag}(U V W 1)$. On the contrary, shortward of $U$ the spectral profile remains constant within the observational errors all the time. We identify the spectral region whose slope is most affected by the gradually increasing opacity with time; it spans between the $U$ and $B$ band (Fig. 9).

Our findings can be explained if line blanketing underwent only small variations and if the UV emitting area tended to shrink during the evolution of SN 2006aj over the time interval under investigation. Moreover, if the underlying source of emission beneath the layer producing the opacity in the UV spectral region is approximated by blackbody (Panagia 2003), then the temperature of this blackbody remains stable during our observing series, because the decrease in the blackbody temperature would also be reflected in the changing slope of the spectral energy distribution in this region. This would lead to reddening of the color indices in this region. However, this is not observed. Further time-dependent models of the spectra and color indices of this asymmetric explosion using realistic opacities and their distribution in velocity space (Foley et al. 2009; Fisher at al. 1997) for the individual spectral regions are needed to reproduce the observed evolution of the colors.

\section{Conclusion}

GRB 060218 is a particularly important event because it is certain to coincide with an SN. We showed in this case that the photometric study of SN features in the light curves of OAs of GRBs is more efficient when it considers the color indices, not just the light curves alone. We showed the power of the method of the color indices that uses the commonly available multiband photometry. The limitation of the photometric method is the investigation of $\mathrm{SNe}$ in the nebular phase when the light of the $\mathrm{SN}$ is dominated by strong emission lines. This nebular phase begins more than two months after $T_{0}$. In the preceding photospheric phase, for which most observations of OAs are carried out, the optical and UV magnitudes obtained in the photometric filters are representative of the continuum light. Not only the color indices of the $\mathrm{SN}$ at a given time, but also their evolution in time appear to be important for its classification and photometric study. Obtaining the dense data series is therefore very important. Using this method we succeeded in analyzing the environments in which the individual components of the afterglow of GRB 060218 occurred. The colors in the cosmic UV band represent the important extension of this method to another spectral region. Our approach is also generally important for investigating the numerous faint OAs for which obtaining spectra with good $\mathrm{S} / \mathrm{N}$ ratio at phases when the $\mathrm{SN}$ light significantly contributes is often impossible. The method of the color indices may also be effectively applied in the ESA Gaia project, especially in confirmation of detected OAs of GRBs without any available trigger in the gamma-ray region.

Acknowledgements. This study was supported by the grant 205/08/1207 provided by the Grant Agency of the Czech Republic and the project ESA PECS 98058 Gaia. G. Pizzichini acknowledges financial support as part of ASI contract $\mathrm{I} / 088 / 06 / 0$

\section{References}

Arnett, W. D. 1979, ApJ, 230, L37

Arnett, W. D. 1982, ApJ, 253, 785

Bloom, J. S., Kulkarni, S. R., Djorgovski, S. G., et al. 1999, Nature, 401, 453 Blustin, A. J. 2007, RSPTA, 365, 1263

Brown, P. J., Roming, P. W. A., vanden Berk, D. E., et al. 2007, in Supernova 1987A: 20 Years After: Supernovae and Gamma-Ray Bursters, AIP Conf. Proc., 937, 386

Brown, P. J., Holland, S. T., Immler, S., et al. 2009, AJ, 137, 4517

Campana, S., Mangano, V., Blustin, A. J., et al. 2006, Nature, 442, 1008 Cusumano, G., Barthelmy, S., Gehrels, N., et al. 2006, GCN Circ., 4775 Della Valle, M., Malesani, D., Bloom, J. S., et al. 2006, ApJ, 642, L103 Ferrero, P., Kann, D. A., Zeh, A., et al. 2006, A\&A, 457, 857 Fisher, A., Branch, D., Nugent, P., et al. 1997, ApJ, 481, L89 Fitzpatrick, E. L., \& Massa, D. 2007, ApJ, 663, 320

Foley, R. J., Papenkova, M. S., Swift, B. J., et al. 2003, PASP, 115, 1220 Foley, R. J., Chornock, R., Filippenko, A. V., et al. 2009, AJ, 138, 376 Galama, T. J., Vreeswijk, P. M., van Paradijs, J., et al. 1998, Nature, 395, 670 Gal-Yam, A., Poznanski, D., Maoz, D., et al. 2004, PASP, 116, 597 Gehrels, N. 2004, NewAR, 48, 431

Ghisellini, G., Ghirlanda, G., \& Tavecchio, F. 2007, MNRAS, 375, L36 Gorosabel, J., Larionov, V., Castro-Tirado, A. J., et al. 2006, A\&A, 459, L33 Iwamoto, K., Nomoto, K., Hoflich, P., et al. 1994, ApJ, 437, L115 Karp, A. H., Lasher, G., Chan, K. L., et al. 1977, ApJ, 214, 161 Kocevski, D., Modjaz, M., Bloom, J. S., et al. 2007, ApJ, 663, 1180 Li, L.-X. 2008, MNRAS, 388, 603

Maeda, K., Mazzali, P. A., \& Nomoto, K. 2006, ApJ, 645, 1331 Maeda, K., Kawabata, K., Tanaka, M., et al. 2007, ApJ, 658, L5 Maund, J. R., Wheeler, J. C., Patat, F., et al. 2007, A\&A, 475, L1 Mazzali, P. A., Deng, J., Maeda, K., et al. 2002, ApJ, 572, L61 Mazzali, P. A., Deng, J., Nomoto, K., et al. 2006, Nature, 442, 1018 Mirabal, N., Halpern, J. P., An, D., et al. 2006, ApJ, 643, L99 Nakano, S., Kushida, R., Kushida, Y., et al. 2002, IAUC, 7810, 1 Panagia, N. 2003, LNP, 598, 113

Pian, E., Mazzali, P. A., Masetti, N., et al. 2006, Nature, 442, 1011 Poole, T. S., Breeveld, A. A., Page, M. J., et al. 2008, MNRAS, 383, 627 Poznanski, D., Avishay, G.-Y., Maoz, D., et al. 2002, PASP, 114, 833 Richmond, M. W., van Dyk, S. D., Ho, W., et al. 1996, AJ, 111, 327 Soffitta, P., Feroci, M., Piro, L., et al. 1998, IAU Circ., 6884

Sollerman, J., Jaunsen, A. O., Fynbo, J. P. U., et al. 2006, A\&A, 454, 503 Sonbas, E., Moskvitin, A. S., Fatkhullin, T. A., et al. 2008, AstBu, 63, 228 Šimon, V., Hudec, R., Pizzichini, G., et al. 2001, A\&A, 377, 450

Šimon, V., Hudec, R., Pizzichini, G., et al. 2004a, Gamma-Ray Bursts: 30 Years of Discovery: Gamma-Ray Burst Symposium, ed. E. E. Fenimore, \& M. Galassi (Melville, NY: AIP), AIP Conf. Proc., 727, 487

Šimon, V., Hudec, R., \& Pizzichini, G. 2004b, A\&A, 427, 901

Šimon, V., Hudec, R., \& Pizzichini, G. 2006, in Proceedings of Swift and GRBs: Unveiling the Relativistic Universe, Il Nuovo Cimento C, 121 B, 1583

Šimon, V., Pizzichini, G., \& Hudec, R. 2009, Gamma-ray Burst: Sixth Huntsville Symposium, AIP Conf. Proc., 1133, 247

Šimon, V., Hudec, R., \& Pizzichini, G. 2010, Mem. S. A. It., 81, 356

Tinney, C., Stathakis, R., Cannon, R., et al. 1998, IAU Circ., 6896

Valenti, S., Benetti, S., Cappellaro, E., et al. 2008, MNRAS, 383, 1485

Waxman, E., \& Draine, B. T. 2000, ApJ, 537, 796

Wiersema, K., Savaglio, S., Vreeswijk, P. M., et al. 2007, A\&A, 464, 529

Woosley, S. E., Eastman, R. G., \& Schmidt, B. P. 1999, ApJ, 516, 788

Yoshii, Y., Tomita, H., Kobayashi, Y., et al. 2003, ApJ, 592, 467 